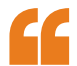

the use of

distally located

exit sites to

enforce spatial

separation

of template

and nascent

strands might

represent

a universal

mechanism for

sNSV genome replication

\title{
Copying the bunyavirus genome
}

Segmented negative-strand RNA viruses (sNSVs) use an RNAdependent RNA polymerase (RdRp) to transcribe and replicate their genomes. For orthomyxoviruses (such as influenza virus), the RdRp consists of a heterotrimeric complex, whereas in bunyaviruses (such as La Crosse orthobunyavirus (LACV)), the RdRp consists of a single protein, termed the L protein, that lacks sequence homology with the orthomyxovirus counterpart. Thus, although the structure of the influenza virus RdRp has been solved, whether this structure can be used to model viral RNA (vRNA) replication by other sNSVs has been unclear. Gerlach et al. now report atomic resolution structures of the LACV L protein, which suggest a new model for sNSV genome replication and indicate that orthomyxovirus and bunyavirus RdRps have a common evolutionary origin.

$\mathrm{X}$-ray crystal structures of the $\mathrm{L}$ protein complexed with short oligonucleotides derived from the LACV promoter (the $5^{\prime}$ and $3^{\prime}$ ends of the vRNA, which are highly complementary) reveal that the overall architecture of the L protein is strikingly similar to the influenza virus RdRp heterotrimer. In influenza virus, the $\mathrm{PB} 1$ protein, which contains the polymerase active site, is enclosed on either side by the $\mathrm{PB} 2$ protein and the carboxyl terminus of the PA protein (PA-C) to form a globular core; similarly, the polymerase domain of the L protein is surrounded by PB2-like and PA-Clike domains. However, an important difference was observed in the vRNA binding lobe (vRBL), which interacts with the promoter: although an arch that binds the $5^{\prime}$ end of the vRNA is present in the lobes of both enzymes, the LACV vRBL contains an additional clamp that binds the 3 ' end of the vRNA. Cryo-electron microscopy showed that this clamp is flexible and the authors speculate that it might reposition the 3' end of the vRNA into a pre-initiation conformation. Furthermore, following binding by the $5^{\prime}$ end of the vRNA, a conformational change is induced that seems to activate polymerase activity, which suggests allosteric regulation by the $5^{\prime}$ end of the vRNA.

The L protein also contains four tunnels that are arranged in a similar configuration to the influenza virus RdRp; the L protein structure revealed one

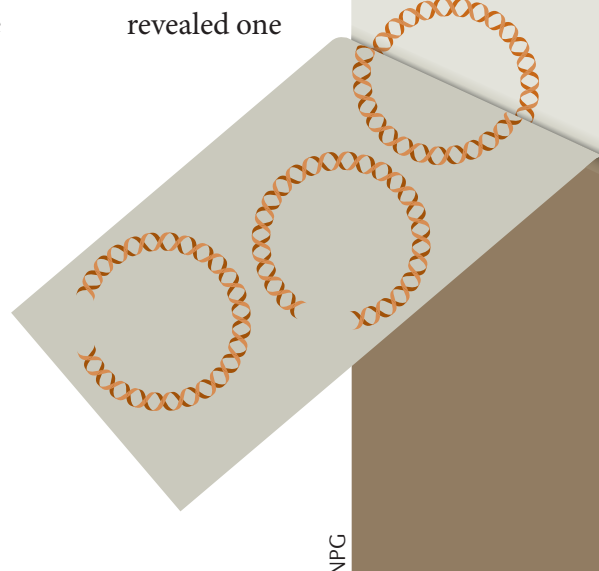

of these to be a candidate exit tunnel for the vRNA template strand with an exit site distal to the exit site of the nascent strand. This positioning would ensure spatial segregation of the two strands following RNA synthesis to prevent the formation of an RNA duplex that might impede processivity of the enzyme. Furthermore, the exit site of the template strand is adjacent to its entry site, which enables it to reintegrate into the viral ribonucleoprotein at or near its original location.

Based on these data, the authors propose a model in which vRNA binding induces conformational changes that initiate replication. Then, during synthesis, the spatial arrangement of exit tunnels segregates the nascent and template strands and facilitates reincorporation of the template strand into the ribonucleoprotein. Given the structural similarity between bunyavirus and influenza virus RdRps, the authors propose that the use of distally located exit sites for the template and nascent strands might represent a universal mechanism for sNSV genome replication.

Naomi Attar

ORIGINAL RESEARCH PAPER Gerlach, P. et al. Structural insights into Bunyavirus replication and its regulation by the vRNA promoter. Cell http:// dx.doi.org/10.1016/j.cell.2015.05.006 (2015) 